Pub. Mat. UAB

$N^{\circ} 25$, Juny 1981

\title{
ON THE NUMBER OF PERIODIC ORBITS OF CONTINUOUS \\ MAPPINGS OF THE INTERVAL
}

\section{Jaume Lijbre and Agusti Reventós}

Secció de Matenàtiques, Facultat de Ciències, Universitat Autònoma de Barcelona, Bellaterra, Barcelona, Spain.

Rebut ]'1 de Juny de? 1981

Abstract. Let $f$ be a continuous map of a closed interval into itself, and let $P(f)$ denote the set of positive integers $k$ such that $f$ has a periadic point of period $k$. Consider the following ordering of positive integers: $3,5,7, \ldots, 2.3,2.5,2.7, \ldots, 4.3,4.5,4.7, \ldots, 8,4,2,1$. Sarkovskii"s theorem states that if $n \in P(f)$ and $m$ is to the right of $n$ in the above ordering then $m \in P(f)$. We may ask the following question: if $n \in P(f)$ and $m$ is to the right of $n$ in the sbove ordering what can be said about the number of periodic orbits of $f$ of periad $m$ ?. We give the answer to this question if $n$ is either odd or $a$ power of 2 . 


\section{Introduction}

This paper is concerned with the periadic arbits of continuous mappings of the interval into itself. Let I denote a closed interval on the real line and let $C^{D}(I, I)$ denote the space of continuous maps of $I$ into itself. For $f \in C^{0}(I, I)$, let $P(f)$ denate the set of pasitive integers $k$ such that $f$ has (at least) a periodic point of period $k$ (see section 2 for definition). One may ask the following question: If $k \in P(f)$, what other integers must be elements of $P(f)$ ?.

This question is answered by a theorem of Sarkovski.i. Consider the following ordering of the set of positive integers $\mathrm{N}$ :

$$
3,5,7, \ldots, 2.3,2.5,2.7, \ldots, 4.3,4.5,4.7, \ldots, 3,4,2,1 \text {. }
$$

Thus, in this ordering the smallest element of $N$ is 3 and the greatest is 1. Sarkovskij's theorem states that if $n \in P(f)$ and $m$ is to the right of $n$ in the above ordering (Sarkovskii ordering) then there is at Ieast one periodic orbit ar period m (see[2] or [3]). Furinermore, if $\mathrm{m}$ is to the left of $\mathrm{n}$ in the Sarkovskii ordering, then there is a $\operatorname{map} f \in C^{a}(I, I)$ with $\pi \in P(f)$ and $m \notin P(f)$.

For $f \in C^{D}(I, I)$, let $N(f, m)$ denote the number of periodic orbits of $f$ of period m. In this paper, we ask the following question: If $n \in P(f)$ and $m$ is to the right of $n$ in the Sarkovskii ordering, what can be said about $N(f, m)$ ?. Dur main result is the following.

Theorem $A$. Let $f \in C^{0}(I, I)$ and let $n$ denote the minimum of $P(f)$ in the Sarkovskii ordering. Suppose $n$ is add, $n>1$ and $m$ is to the right of $n$ in the Sarkovskii ordering. Then the following hold.

(i) There is an integer $N_{n}(m)$ (easily computable, see section 3 ) such that $N(f, m) \geqslant N(m)$.

(ii) There is a map $g \in C^{\circ}(I, I)$ such that $P(g)=P(f)$ and $N(g, m)=N_{n}(m)$. 
Note, for example, that if $f \in C^{0}(I, I)$ and $3 \in P(f)$, then $f$ has at least $N_{3}(m)$ periodic orbits of period $m$. We have campute $N_{3}(m)$ and $N_{5}(m)$ for $m=1,2, \ldots, 50$ in Tables $I$ and $I I$, respectively (for details see section 3). We remark that Sarkovskii's theorem only says $N_{n}(m) \geqslant 1$. Proposition B. Let $f \in C^{0}(I, I)$ and let $n$ denote the minimum of $P(f)$ in the Sarkovskii ordering. Suppose $n$ is a power of 2 and $m$ is to the right of $n$ in the Sarkovskij ordering. Then the integer $N_{n}(m)$ which satisfies conditions (i) and (ii) of Theorem $A$ is the unity.

Proposition $\mathrm{B}$ follaws immediately from the fact that for each power of 2 , let $2^{r}$, there is a map $f \in C^{D}(I, I)$ such that $P(f)=$ $=\left\{1,2,4, \ldots, 2^{r}\right\}$ and $N\left(f, 2^{k}\right)=1$ for $k=0,1, \ldots, r$ (see Lemma 16 of $[1\})$.

In proving Theorem $A$, we use a result of Stefan (see section 2). This result describes how a mapping $f \in C^{\circ}(I, I)$ must act on a periodic orbit $\left\{p_{1}, \ldots, p_{n}\right\}$ of odd period $n>1$, where $n$ is the minimum of off) in the Sarkovskii ordering.

We note the algorithm described in order to compute the integer $N_{n}(m)$ defined in Theorem A (see section 3) can be used for all $n \in P(f)$ not necessarily odd. But we need to know haw $f$ must act on a periodic arbit of $f$ of period $n$. That is, if $\left\{p_{1}, \ldots, p_{n} \mid\right.$ is a periodic orbit of $f$ of period $n$, who is $f\left(p_{i}\right)$ for each $i=1, \ldots, n$ ?.

We are grateful to Ramon Reventós who have helped us in the preparation of this note.

\section{Preliminary definitions and results}

Let $f \in C^{\circ}(I, I)$. For any positive integer $n$, we define $f^{n}$ inductively by $f^{1}=f$ and $f^{n}=f \cdot f^{n-1}$. We let $f^{a}$ denate the identity map of $I$.

Let $p \in I$. We say $p$ is a fixed point of $f$ if $f(p)=p$. If $p$ is a fixed point of $f^{n}$, for some $n \in N$, we say $p$ is a periodic point 
of $f$. In this case the smallest element of $\left\{n \in N: f^{n}(p)=p\right\}$ is called the period of $p$.

We define the orbit of $p$ to be $\left\{f^{n}(p): n=0,1,2, \ldots\right.$, If $\rho$ is a periodic point of $f$ of period $n$, we say the orbit of $p$ is $a$ periodic orbit of period $n$. In this case the orbit of $\rho$ cantains exactly $n$ points each of which is a periodic point of period $n$.

We will use the following theorem (see Theorem 2 of Stefan [31).

Theorem 1. Let $f \in C^{\circ}(I, I)$ and let $n$ denote the minimum of $P(f)$ in the Serkouskii ordering. Suppose $n$ is odd and $n>1$. Let $\left\{p_{1}, \ldots, p_{n}\right\}$ be a periodic orbit of period $n$ with $p_{1}<p_{2}<\cdots<p_{n}$. Let $t=(n+1) / 2$. Then either (a) or (b) holds (see (a) in fig. 1 for $n=3,5,7,9$ ):

(a) $f\left(p_{t-k}\right)=p_{t+k+1}$ for $k=0, \ldots, t-2$,

$$
\begin{aligned}
& f\left(p_{t+k}\right)=p_{t-k} \quad \text { for } k=1, \ldots, t-1, \text { and } \\
& f\left(p_{1}\right)=p_{t} .
\end{aligned}
$$

(b) $f\left(p_{t-k}\right)=p_{t+k} \quad$ for $k=1, \ldots, t-1$,

$$
\begin{aligned}
& f\left(p_{t+k}\right)=p_{t-k-1} \quad \underline{f n g} k=0, \ldots, t-2 ; \text { 케 } \\
& f\left(p_{n}\right)=p_{t} .
\end{aligned}
$$
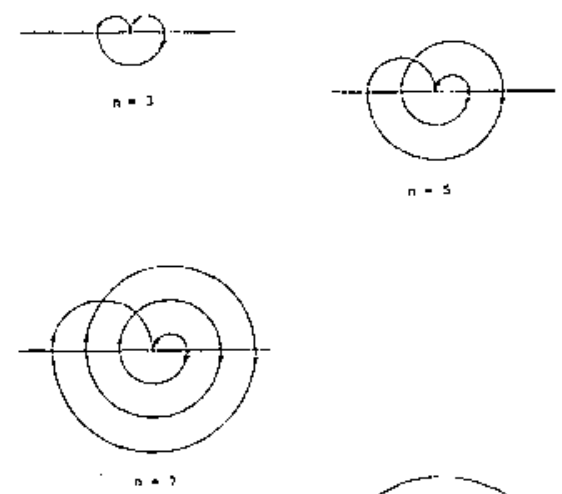

127- 1

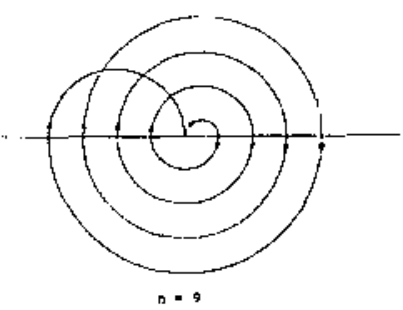




\section{Proof of Theorem $A$}

Let $f=C^{O}(I, I)$ and let $n$ denote the minimum of $P(f)$ in the Sarkovskii ordering. Suppose $n$ is add and $n\rangle\}$. Let $\left\langle p_{1}, \ldots, p_{n}\right\}$ be a periodic orbit of $f$ of period $n$. We can assume that we are in the case (a) of Theorem 1 (the case (b) is similar).

Now, we study the map $g:\left[p_{p}, p_{n}\right] \longrightarrow\left[p_{1}, p_{n}\right]$ defined by $g\left(p_{t-k}\right)=p_{t+k+1}$. for $k=0, \ldots, t-2$,

$g\left(\rho_{t+k}\right)=\rho_{t-k} \quad$ for $k=1, \ldots, t-1$, and

$g\left(\rho_{1}\right)=p_{t}$

where $t=(n+1) / 2$, and on each interval $\left\{p_{i}, p_{i+\uparrow}\right\}, i=1, \ldots, n-1$, assume $g$ is linear (see fig. 2 for $n=3$ and $n=5$ ).

Suppose $m$ is to the right of $n$ in the Sarkovskii ordering. By continuity, $N(f, m) \geqslant N(g, m)$. Let $N_{n}(m)=N(g, m)$. Now, we shall give an algorithm to compute $N_{n}(m)$ and Theorem $A$ will follow. We only describe the algorithm to compute $N_{n}(m)$ for $n=3$ and $n=5$, since for the other values of $n($ odd $)$, it is similar.
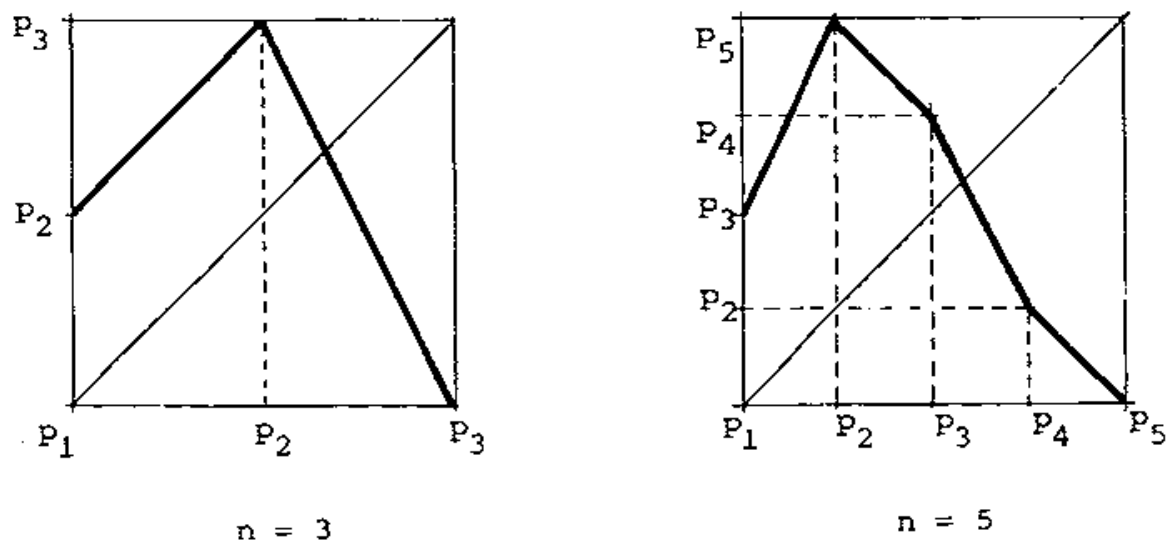

Fig. 2 
Suppose $n=3$. Let $\left\{a_{1}, a_{2}, \ldots, a_{k}(m)\right\}$ denote the set of points of $\left[p_{1}, P_{3}\right]$ where $g^{m}$ has a maximum or a minimum. It is easy to see that $q_{1}=p_{1}, a_{k(m)}=p_{3}, p_{2} \in\left\{a_{1}, \ldots, a_{k(m)}\right\}, g^{m}\left(\left\{q_{1}, \ldots, a_{k}(m)\right\}\right)=$ $=\left\{p_{1}, p_{2}, p_{3}\right\}$ and $g^{m}\left(\left[q_{i}, q_{i+1}\right]\right)$ is either $\left[p_{2}, p_{3}\right]$ or $\left[p_{1}, p_{3}\right]$ for each $i=1, \ldots, k(m)-1$.

Let $a_{23}(m)$ (respectively $b_{23}(m)$ ) be the number of intervals $\left[q_{i}, q_{i+1}\right] \subset\left[p_{1}, p_{2}\right]$ (respectively $\left.\left[p_{2}, p_{3}\right]\right)$ such that $g^{m}\left(\left[q_{i}, q_{i+1}\right]\right)=$ $=\left[\mathrm{p}_{2}, \rho_{3}\right]$ - Let ${ }_{33}(\mathrm{~m})\left(\right.$ respectively $\left.b_{13}(\mathrm{~m})\right)$ be the number of intervals $\left[q_{i}, q_{i+1}\right] \leftrightharpoons\left[p_{1}, p_{2}\right] \quad$ (respectively $\left.\left[p_{2}, p_{3}\right]\right)$ such that $\left.g^{m}\left(\mid q_{i}, q_{i+1}\right)\right)=$ $=\left[p_{1}, p_{3}\right]$.

From the definition of $\mathrm{g}$ it is clear that

$$
\begin{array}{ll}
a_{123}(1)=1, & a_{13}(1)=0, \\
b_{23}(1)=0, & b_{13}(1)=1,
\end{array}
$$

and

$$
\begin{array}{ll}
a_{23}(m+1)=a_{13}(m), & a_{13}(m+1)=a_{23}(m)+a_{13}(m), \\
b_{23}(m+1)=b_{13}(m), & b_{13}(m+1)=b_{23^{(m)}}\left(b_{13}(m),\right.
\end{array}
$$

for $m=1,2, \ldots$

Since the fixed points of $\mathrm{g}^{\mathrm{m}}$ are the points of the graphic of $g^{m}$ which ere on the diagonal of the square $\left[p_{1}, p_{3}\right] \times\left[p_{1}, p_{3}\right]$, we obtain that $\mathrm{g}^{\mathrm{m}}$ has

$$
a_{13}(m)+b_{23}(m)+b_{13^{(m)}}=\left(\frac{1+\sqrt{5}}{2}\right)^{m}+\left(\frac{1-\sqrt{5}}{2}\right)^{m},
$$

fixed goints. Then it is easy to compute $N_{n}(m)$ for $n=3$ (see Table I). Note the number of fixed points of $\mathrm{g}^{\mathrm{m}}$ is a $F$ ibonacci number.

Now, suppose $n=5$. Let $\left\{a_{1}, \ldots, q_{k(m)}\right\}$ denote the set of points of $\left[\rho_{1}, \rho_{5}\right]$ where $g^{m}$ has a maximum or a minimum. It is easy to see that $\left.q_{3}=p_{1}, q_{k(m)}=p_{5},\left\{p_{2}, p_{3}, p_{4}\right\} \subset\left\{q_{1}, \ldots, q_{k}(m)\right\}, g^{m}\left(j a_{1}, \ldots, q_{k}(m)\right\}\right)=$ $=\left\{p_{1}, p_{2}, p_{3}, p_{4}, p_{5}\right\}$ and $g^{m}\left(\left[q_{i}, q_{i+1}\right]\right)$ is one of the following intervals: $\left[\rho_{3}, p_{5}\right],\left[p_{2}, p_{5}\right],\left[p_{1}, p_{4}\right],\left[p_{1}, p_{5}\right]$, for each $i=1, \ldots, k(m)-1$. 
Let $a_{r s}(m)$ (respectively $\left.b_{r s}(m), c_{r s}(m), d_{r s}(m)\right)$ be the number of intervals $\left[q_{i}, q_{i+1}\right]<\left[p_{1}, p_{2}\right]$ (respectively $\left[p_{2}, p_{3}\right\},\left[p_{3}, p_{4}\right],\left[p_{4}, p_{5}\right]$ ) such that $g^{m}\left(\left[q_{i}, q_{i+1}\right]\right)=\left[p_{r}, p_{s}\right]$. From the definition of $g$ we have that

$$
\begin{aligned}
& a_{35}(3)=1, \quad a_{25}(3)=1, \quad a_{14}(3)=0, \quad a_{15}(3)=0, \\
& b_{35}(3)=1, b_{25}(3)=0, b_{14}(3)=0, b_{15}(3)=0 \text {, } \\
& c_{35}(3)=0, \quad c_{25}(3)=0, \quad c_{14}(3)=0, \quad c_{15}(3)=1 \text {, } \\
& d_{35}(3)=0, \quad d_{25}(3)=0, \quad d_{14}(3)=1, \quad d_{15}(3)=0 \text {, }
\end{aligned}
$$

and

$$
\begin{aligned}
& x_{35^{(m+1)}}=x_{14}(m)+x_{15^{(m)}} \text {, } \\
& \times_{25}(m+1)=x_{14}(m) \text {, } \\
& \times_{14}(m+1)=x_{35}(m) \\
& x_{15^{(m+1)}}=x_{25^{(m)}}+x_{15^{(m)}} \text {. }
\end{aligned}
$$

for $m=3,4, \ldots$ and $x \in\{a, b, c, d\}$.

Because the fixed points of $g^{m}$ are the points of the graphic of $g^{m}$ which are on the diagonal of the square $\left[p_{1}, p_{5}\right] \times\left[p_{1}, p_{5}\right]$, we obtain that $g^{m}$ has

$$
\begin{array}{r}
a_{14}(m)+a_{15}(m)+b_{25}(m)+b_{14}(m)+b_{15}(m)+c_{35}(m)+c_{25}(m)+ \\
c_{14}(m)+c_{15}(m)+d_{35}(m)+d_{25}(m)+d_{15}(m)
\end{array}
$$

fixed. points. Hence it is easy to compute $N_{n}(m)$ for $n=5$ (see Table II). 
Table I

\begin{tabular}{|c|c|}
\hline $\mathrm{m}$ & $\mathrm{N}_{3}(\mathrm{~m})$ \\
\hline 1 & 1 \\
\hline 2 & 1 \\
\hline 3 & 1 \\
\hline 4 & 1 \\
\hline 5 & 2 \\
\hline 6 & 2 \\
\hline 7 & 4 \\
\hline 8 & 5 \\
\hline 9 & 8 \\
\hline 10 & 11 \\
\hline 11 & 18 \\
\hline 12 & 25 \\
\hline 13 & 40 \\
\hline 14 & 58 \\
\hline J. 5 & 90 \\
\hline 16 & 135 \\
\hline 17 & 210 \\
\hline 18 & 316 \\
\hline 19 & 492 \\
\hline 20 & 750 \\
\hline 21 & 1164 \\
\hline 22 & 1791 \\
\hline 23 & 2786 \\
\hline 24 & 4305 \\
\hline 25 & 6710 \\
\hline
\end{tabular}

\begin{tabular}{|c|c|}
\hline$m$ & $\mathrm{~N}_{3}(\mathrm{~m})$ \\
\hline 26 & 10420 \\
\hline 27 & 16264 \\
\hline 28 & 25350 \\
\hline 29 & 39650 \\
\hline 30 & 61967 \\
\hline 31. & 97108 \\
\hline 32 & 152145 \\
\hline 33 & 238818 \\
\hline 34 & 374955 \\
\hline 35 & 589520 \\
\hline 36 & 927200 \\
\hline 37 & 1459960 \\
\hline 38 & 2299854 \\
\hline 39 & 3626200 \\
\hline 40 & 5720274 \\
\hline 41 & 9030450 \\
\hline 42 & 14263078 \\
\hline 43 & 22542396 \\
\hline 44 & 35644500 \\
\hline 45 & 56393760 \\
\hline 46 & 89262047 \\
\hline 47 & 141358274 \\
\hline 48 & 223955235 \\
\hline 49 & 354975428 \\
\hline 50 & 562871705 \\
\hline
\end{tabular}


Table II

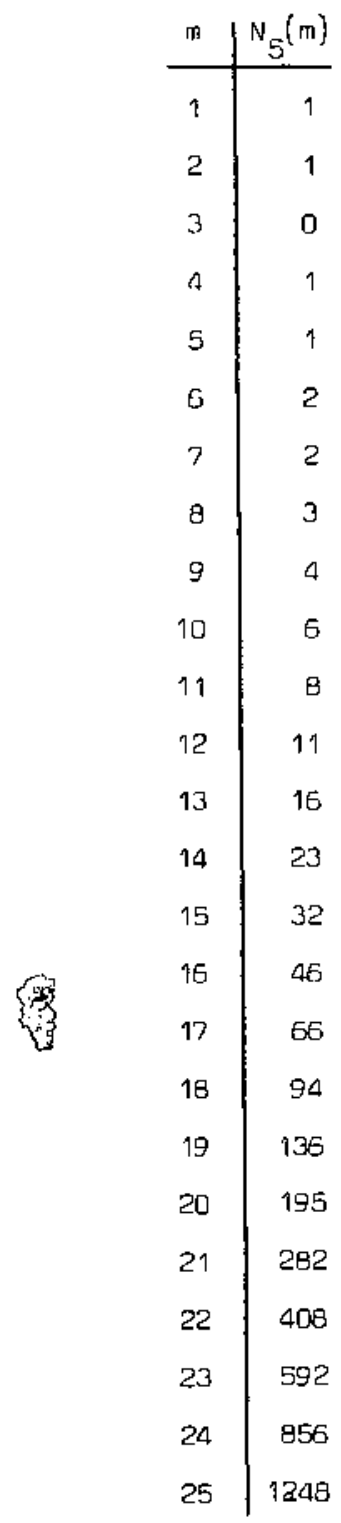

\begin{tabular}{|c|c|}
\hline $\mathrm{m}$ & $N_{5}(m)$ \\
\hline 26 & 1814 \\
\hline 27 & 2646 \\
\hline 28 & 3858 \\
\hline 29 & 5644 \\
\hline 30 & 8246 \\
\hline 31 & 12088 \\
\hline 32 & 17706 \\
\hline 33 & 25992 \\
\hline 34 & 38155 \\
\hline 35 & 56102 \\
\hline 36 & 82490 \\
\hline 37 & 12.1474 \\
\hline 38 & 178902 \\
\hline 39 & 263776 \\
\hline 40 & 380033 \\
\hline 41 & 574304 \\
\hline 42 & 848069 \\
\hline 43 & 1253344 \\
\hline 44 & 1852926 \\
\hline 45 & 2741164 \\
\hline 46 & 4056706 \\
\hline 47 & 6007042 \\
\hline 48 & 8898261 \\
\hline 49 & 13187750 \\
\hline 50 & 19551952 \\
\hline
\end{tabular}




\section{REFERENCES}

1.: Block,L. : The periadic points of Morse-Smale endomorphisms of the circle, Trans. Amer.Math.5oc. 266, 77-88 (1977).

2. Sarkovskii,A.N. : Coexistence of cycles of a continuous map of a line into itself, Lkr. Mat.z 16 , 61-7^ (1964).

3. Stefan,P. : A theorem of Sarkovskii on the existence of periodic orbits of continuous endomorphisms of the real line, Comm.Math.Phys. $54,237-248$ (1977). 